\title{
INVESTIGATION OF DEFECTS IN AMORPHOUS SILICON FILMS USING POSITRON ANNIHILATION
}

\author{
Yang-Fang CHEN, Chia-Chi WANG, Poh-Kun TSENG \\ Physics Department, National Taiwan University, Taipei, Taiwan, ROC \\ and \\ Jun-Tseng LUE \\ Physics Department, National Tsing-Hua University, Hsin-Chu, Taiwan, ROC
}

Received 23 March 1988; revised manuscript received 15 November 1988; accepted for publication 25 November 1988

Communicated by J.I. Budnick

\begin{abstract}
Positron lifetime studies have been carried out on amorphous silicon films prepared by sputtering. Isochronal annealing at different temperatures has been investigated for understanding the microstructure such as vacancies and microvoids. The shortest lifetime component is attributed to the annihilation with the bulk electrons in the crystalline substrate. Information concerning the effective volume of the dominant vacancy type defect, quadrivacancy, in the films is obtained from the study of the middle lifetime component. The appearance of the longest lifetime component $(t \approx 2 \mathrm{~ns})$ suggests the existence of large microvoids in the films.
\end{abstract}

Although the technical use of amorphous semiconductors (notably $\mathrm{Si}$ ) has prompted considerable interest in recent years, the investigation of amorphous silicon has mainly been done by electrical conductivity measurements, optical absorption, electron spin resonance, and also by electron microscopy [1], etc. However, none of these methods leads in itself to a determination of very small vacancy-type defects! Positron annihilation depends upon the electron density at the site of the positron and can be used to characterize the nature of the positrontrapping site. This technique has been successfully used to study the defects in materials. In the present paper, we report the results of positron annihilation studies on relatively thick $(\approx 10 \mu \mathrm{m})$ amorphous silicon (a-Si) films. Through this study it is possible to infer the existence of clusters of vancancies and microvoids in a-Si. The variation of annihilation parameters with the annealing temperature of the films is explained satisfactorily. Even though some positron annihilation experiments on a-Si have been published [2-9], to our knowledge there is still no definite conclusion.

All a-Si samples were rf-sputtered onto polished ptype single crystal silicon wafers. The film thickness is about $10 \mu \mathrm{m}$ which was achieved by sputtering an n-type $(10 \Omega \mathrm{cm})$ single crystal silicon wafer in an argon atmosphere. The deposition conditions were as follows: Prior to sputtering pressure, $1 \times 10^{-6}$ Torr, during sputtering pressure, $1 \times 10^{-2}$ Torr; substrate temperature, $300 \mathrm{~K}$ (the substrate holder is water cooled); and RF power, $60 \mathrm{~W}$. The infrared absorption spectrum of a-Si as measured by a Perkin-Elmer (model $710 \mathrm{~B}$ ) double-beam spectrometer shows no detectable Si-H stretching mode occurring at 2100 $\mathrm{cm}^{-1}$. Thus, we presume that the effect of hydrogen on the positron annihilation measurements can be ignored.

The positron source was a $1 \mu \mathrm{Ci} \mathrm{Na}{ }^{22} \mathrm{Cl}$ source deposited on a mica foil. The counting rate was 40 counts per second and each spectrum contained $2 \times 10^{6}$ counts. The full width at half maximum 
(FWHM) of the resolution function was about 450 ps. Analysis of the lifetime spectra was done using the POSITRONFIT extended program [10]. The resolution function was approximated by one gaussian. There was no source correction which was expected to be of little importance in this experiment. All positron measurements were carried out at room temperature. The isochronal annealing was performed in an infrared radiation furnace. Each isochronal annealing step lasted for $30 \mathrm{~min}$. During the annealing and natural cooling processes, samples were placed in a quartz tube with a flow of dry nitrogen.

The lifetime spectra were fitted by three components with intensities and lifetimes designated as $\left(I_{1}\right.$, $\left.t_{1}\right),\left(I_{2}, t_{2}\right)$, and $\left(I_{3}, t_{3}\right)$, respectively. The shortest lifetime has a value of $208 \pm 10$ ps which does not change with increasing the annealing temperature up to $700^{\circ} \mathrm{C}$ as shown in fig. 1 . This lifetime was not fixed during the analyses, thus serving as a check on the individual measurement, and proved independent of annealing temperature. $t_{1}$ has been attributed
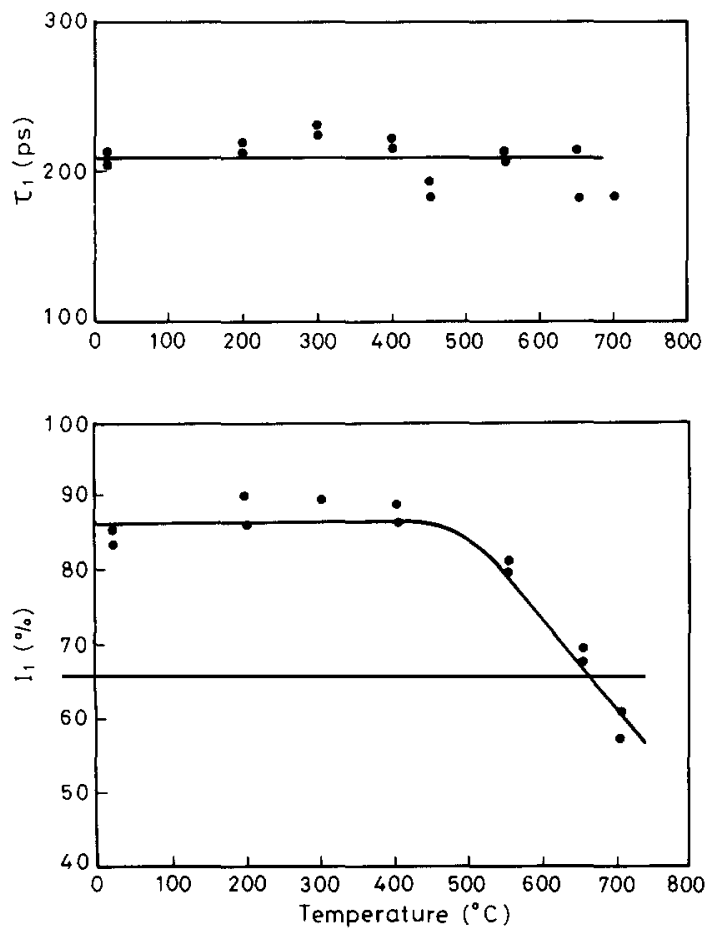

Fig. 1. Lifetime $t_{1}$ and the corresponding intensity versus isochronal annealing temperature. The lines drawn through the data are used to guide the eye only. to the positrons trapped in shallow traps in a-Si by He et al. [2] and Bhide et al. [3]. We believe that this lifetime should correspond to the annihilation of positrons with the bulk electrons in the crystalline silicon, where positrons have penetrated through the amorphous film and into the crystalline silicon. Since Dannefaer et al. [4] have performed a positron experiment on a-Si having a thickness of only $1 \mu \mathrm{m}$, no response except for the $t_{1}$ bulk lifetime could be detected. Thus, $t_{1}$ cannot be due to the annihilation of positrons in an amorphous film or on the surface between the a-Si and the c-Si, otherwise $t_{2}$ should also be observed in the experiment of Dannefaer et al. [4]. The decrease in $I_{1}$ at $450^{\circ} \mathrm{C}$ as shown in fig. 1 is the result of the increase of positrons trapped in the a-Si defects which will be discussed below.

The relation between the middle component $t_{2}$ and annealing temperature is shown in fig. 2 . This lifetime has a constant value of $420 \pm 20$ ps below about $450^{\circ} \mathrm{C}$, after which temperature it starts to decrease. The corresponding intensity $I_{2}$ component is also shown in fig. 2 . It keeps constant below $450^{\circ} \mathrm{C}$, while above that temperature $I_{2}$ increases sharply. In an earlier paper [11] the lifetime in divacancies and
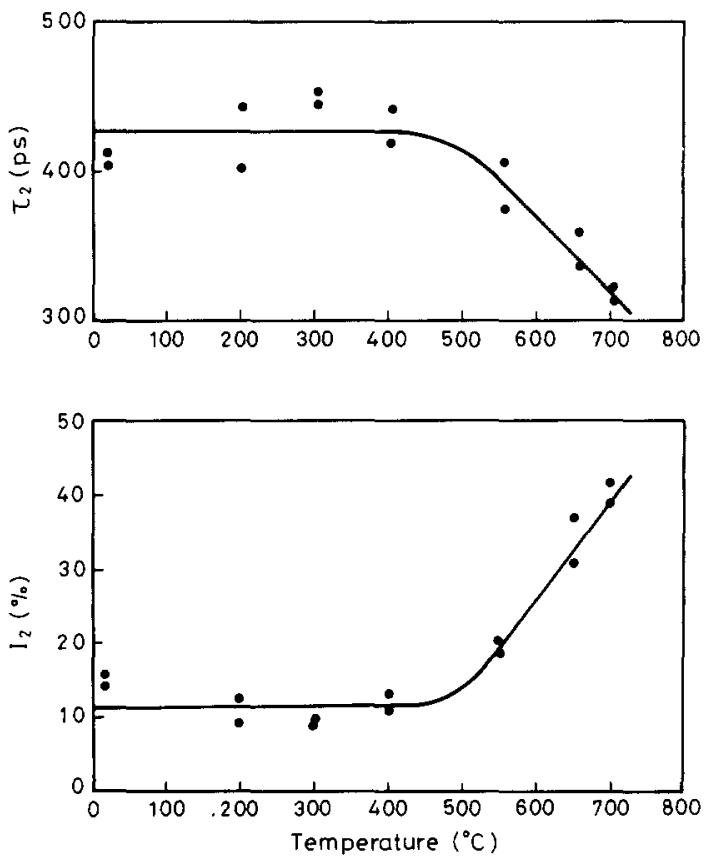

Fig. 2. Lifetime $t_{2}$ and the corresponding intensity versus isochronal annealing temperature. 
quadrivacancies was determined to be $325 \pm 20$ and $435 \pm 30 \mathrm{ps}$, respectively, while for monovancies, Fuhs et al. [12] determined a lifetime of $266 \pm 10$ ps. Comparing these results, we can therefore say that the effective volume of the most dominant vacancy type defect in a-Si is quadrivacancy when the annealing temperature is below $450^{\circ} \mathrm{C}$. There are two important factors which will influence the $t_{2}$ component at higher annealing temperature. First, Fang et al. [13] have performed the gas emission experiments which show that argon escapes around $400^{\circ} \mathrm{C}$. If the argon is released, the number of vacancies will increase and hence the increase in $I_{2}$ at $450^{\circ} \mathrm{C}$ could be a result of the release of argon from the silicon film. Thus, the decrease of the annihilation intensity $I_{1}$ of positrons with the bulk electrons in the crystalline silicon at $450^{\circ} \mathrm{C}$ as shown in fig. 1 is the result of the increase of positrons trapped in the a-Si vacancies which reduces the number of positrons penetrating into the c-Si. The continuous decrease in $t_{2}$ after $450^{\circ} \mathrm{C}$ can be explained by the fact that after argon escaping the annealing causes the mean defect volume to reduce such that the mean result of the $t_{2}$ component decreases. Second, the a-Si films are known to crystallize in the temperature range $600-$ $700^{\circ} \mathrm{C}$. It is thus reasonable for larger vacancies to break down to become smaller vacancies at crystallization. Thus, the crystallization will also increase the intensity $I_{2}$ and decrease the lifetime $t_{2}$.

The longest lifetime $t_{3}$ and the correspondng intensity $I_{3}$ are shown in fig. 3 . It can be seen that $t_{3}$ keeps constant and has a value of $2 \mathrm{~ns}$ below $400^{\circ} \mathrm{C}$, increases with temperature up to $3 \mathrm{~ns}$ at $650^{\circ} \mathrm{C}$, and then sharply drops down. $I_{3}$ decreases below $400^{\circ} \mathrm{C}$, increases with temperature until $650^{\circ} \mathrm{C}$, and then sharply drops down. This component can possibly arise from the positronium formation in the microvoid. He et al. [2] and Bhide et al. [3] have carried out angular correlation experiments of positron annihilation radiation (ACPAR) in a-Si, and confirmed the formation of positronium which they supposed is formed in microvoids. Their origin has been attributed to a lack of surface mobility of the deposited atoms and to a mechanism which relieves the strain in the amorphous network [14]. The decrease in $I_{3}$ below $400^{\circ} \mathrm{C}$ may be due to the rearrangement of the amorphous network which will decrease the number of microvoids. When the annealing
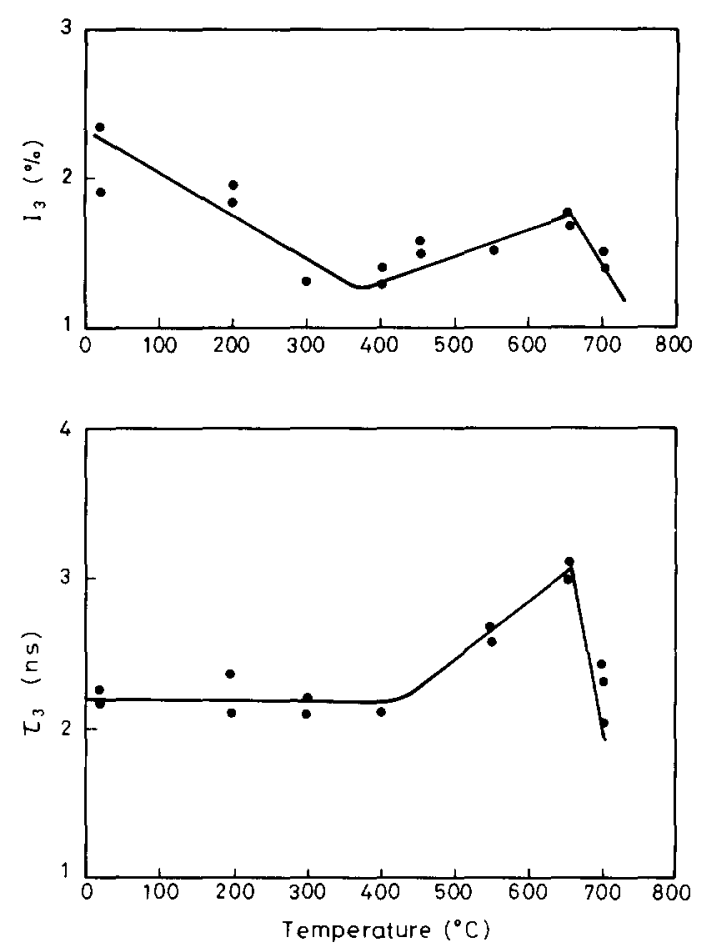

Fig. 3. Lifetime $t_{3}$ and the corresponding intensity versus isochronal annealing temperature.

temperature is above $400^{\circ} \mathrm{C}$, the trapping centers (divacancies and quadrivacancies) aggregate to form microvoids or migrate to connect with the microvoid and increase the size of the microvoid. Thus, $I_{3}$ and $t_{3}$ both increase. When the films start to crystallize at $650^{\circ} \mathrm{C}$, the microvoids break down and $I_{3}$ and $t_{3}$ decrease sharply.

Positron annihilation studies in a-Si films formed by sputtering have shown that the effective volume of the most dominant vacancy type defect is quadrivacancy when the annealing temperature is below $450^{\circ} \mathrm{C}$, and that there exist microvoids. The variation of the intensity and lifetime of the three components in the lifetime spectra $I_{1}$ and $t_{1}\left(t_{1} \approx 210 \mathrm{ps}\right.$ due to annihilaton with bulk electrons in the crystralline substrate), $I_{2}$ and $t_{2}\left(t_{2} \approx 420\right.$ ps due to trapping in quadrivacancies $)$, and $I_{3}$ and $t_{3}\left(t_{3} \approx 2\right.$ ns due to formation of positronium in microvoids) with the isochronal annealing temperature has helped in understanding the a-Si microstructure. The positron annihilation technique has thus proved a useful tool to characterize amorphous semiconductors. 
We thank Dr. Y.C. Jean of the Department of Physics, University of Missouri-Kansas City, for providing the POSITIRONFIT extended program, and Dr. G.J. Jan for help in the isochronal annealing. This work was supported in part by the National Science Council of the Republic of China.

\section{References}

[1] P.K. Willardson and A.C. Beer, eds., Semiconductors and semimetals, Vol. 21 (Academic Press, New York, 1984).

[2] YJ. He, M. Hasegawa, R. Lee and S. Berko, Phys. Rev. B 33 (1986) 5924

[3] V.G. Bhide, R.O. Dusane, S.V. Raharshi, A.D. Shaligram and S.K. David, J. Appl. Phys. 62 (1987) 108.

[4] S. Dannefaer, D. Kerr and B.G. Hogg, J. Appl. Phys. 54 (1983) 155.
[5] H.E. Schaefer, R. Würschum, R. Schwarz, D. Slobodin and S. Wagner, Appl. Phys. A 43 (1987) 295.

[6] S. Dannefaer, P. Mascher and D. Kerr, Appl. Phys. A 43 (1987) 91.

[7] H.E. Schaefer and R. Würschum, Appl. Phys. A 40 (1986) 145.

[8] A.L. Jung, Y.H. Wang and G. Liu, J. Non-Cryst. Solids 74 (1985) 19.

[9] A.L. Jung, Y.H. Wang and G. Liu, J. Non-Cryst. Solids 77 (1985) 88, 221.

[10] P. Kirckegarrd and C.M. Eldrup, Comput. Phys. Commun. 7 (1974) 401.

[11] S. Dannefaer, G.W. Dean, D.P. Kerr and B.G. Hogg, Phys. Rev. B 14 (1976) 2709.

[12] W. Fuhs, V. Holzhauer, S. Mantl, F.W. Richter and R. Strum, Phys. Stat. Sol. (b) 89 (1978) 69.

[13] C.J. Fang, I. Ley, H.R. Shanks, K.J. Gruntz and M. Cardona, Phys. Rev. B 22 (1980) 6104.

[14] G.A.N. Connell and J.R. Pawlik, Phys. Rev. B 13 (1976) 787. 